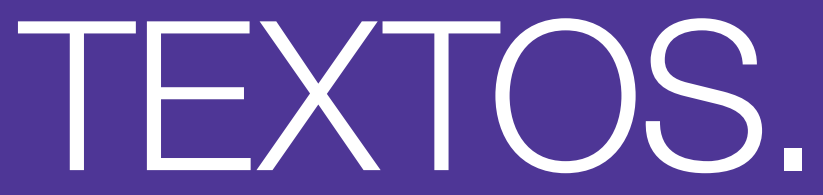

\title{
Revista Internacional de
}

Aprendizaje y Cibersociedad 



\section{Revista Internacional de Aprendizaje y Cibersociedad}

VOLUMEN 18 NÚMERO 12014 
TEXTOS. REVISTA INTERNACIONAL DE APRENDIZAJE Y CIBERSOCIEDAD http://aprendizaje-cibersociedad.com/publicaciones/revista

Publicado en 2014 en Madrid, España por Common Ground Publishing España S.L. www.commongroundpublishing.es

ISSN: $1577-3760$

(c) 2014 (revistas individuales), el autor (es)

@ 2014 (selección y material editorial) Common Ground Publishing España

Todos los derechos reservados. Aparte de la utilización justa con propósitos de estudio, investigación, crítica o reseña como los permitidos bajo la pertinente legislación de derechos de autor, no se puede reproducir mediante cualquier proceso parte alguna de esta obra sin el permiso por escrito de la editorial. Para permisos y demás preguntas, por favor contacte con <soporte@commongroundpublishing.com>.

TEXTOS. Revista Internacional de Aprendizaje y Cibersociedad es revisada por expertos y respaldada por un proceso de publicación basado en el rigor y en criterios de calidad académica, asegurando así que solo los trabajos intelectuales significativos sean publicados. Acepta artículos en español y portugués.

Composición tipográfica en Common Ground Markup Language utilizando un sistema CGPublisher de composición tipográfica multicanal http://www.commongroundpublishing.com/software/ 


\section{EDITORES}

José Francisco Álvarez, Universidad Nacional de Educación a Distancia (UNED), España

Nilton Bahlis Dos Santos, Nucleo de Experimentação de Tecnologias Interativas Next/Icict/Fiocruz e do Clube do Futuro, Brasil

Daniel Domínguez Figaredo, Universidad Nacional de Educación a Distancia (UNED), España María del Carmen Gil Ortega, University of the West of England, Reino Unido

\section{CONSEJO EDITORIAL}

Ana Abreu, Universidade Federal de São Carlos (UFSCar), Brasil Manuel Acevedo Ruiz, Consultor independiente, Argentina

Francisco Álvarez, Universidad Nacional de Educación a Distancia (UNED), España

Elisenda Ardevol, Universitat Oberta de Catalunya (UOC), España

Nilton Bahlis Dos Santos, Nucleo de Experimentação de Tecnologias Interativas Next/Icict/Fiocruz e do Clube do Futuro, Brasil

Françesc Balagué, Observatorio para la Cibersociedad, España

Roberto Balaguer Prestes, Instituto Universitario CLAEH, Uruguay

Bibiana Apolonia del Brutto López, Universidad de Buenos Aires, Argentina

Mario Brun, Didáctica Innovación y Multimedia - Universitat Autònoma de Barcelona, España

Eurídice Cabañes Martínez, ARSGAMES (Game Art \& Game Studies), España

Igor Calzada, Ikerbasque, España

Jesús Carreras, Designit, España

Francisco Javier Cortázar Rodríguez, Universidad de Guadalajara, México

Renato Dias Baptista, Universidade Estadual Paulista (UNESP), Brasil

Daniel Domínguez Figaredo, Universidad Nacional de Educación a Distancia (UNED), España Javier Echeverria, Ikerbasque, España

Nicole Etchevers Goijberg, Herder Editorial, S.L. / Observatorio para la CiberSociedad, España

Ricard Faura i Homedes, Secretaria de Telecomunicacions i Societat de la Informació - Generalitat de

Catalunya, España

Tiberio Feliz, Universidad Nacional de Educación a Distancia (UNED), España

Liliana Edith Ferrari, U. Autónoma de Barcelona / Univ. Nacional de Buenos Aires, Argentina

Inma Fouce, Observatorio para la Cibersociedad, España

Leonarda García Jiménez, Universidad Católica Murcia, España

Fernando Garrido Ferradanes, Observatorio para la CiberSociedad, España

Inés Gil, Universidad Nacional de Educación a Distancia (UNED), España

Mari Carmen Gil Ortega, University of the West of England, Reino Unido

Edgar Gómez, Universitat Oberta de Catalunya (UOC), España

Adriana Goñi Godoy, Universidad de Chile, Chile

Manuel Gromaz Campos, Consellería de Educación - Xunta de Galicia, España

Maritza Guaderrama, Designit, España 
Octavio Islas, Proyecto Internet, Tecnológico de Monterrey, México

Tíscar Lara, Escuela de Organización Industrial (EOI), España

Claudia A. Lerma Noriega, Ins. Tecnológico y de Estudios Superiores de Monterrey, México

Maximiliano Martín Vicente, Universidade Estadual Paulista (UNESP), Brasil

Lisete Maria Massulini Pigatto, Aluna Doutorado pela Universidade Tecnologica Intercontinental do

Paraguai, Brasil

Joan Mayans i Planells, ACC1Ó / Observatorio para la CiberSociedad, España

Guillem Mundet i Genís, Oficina de Patrimoni Cultural, Diputació de Barcelona, España

Sara Osuna Acedo, Universidad Nacional de Educación a Distancia (UNED), España

Ismael Peña, Universitat Oberta de Catalunya (UOC), España

Víctor Manuel Pérez Martínez, Universidad de La Laguna, España

Jimmy Rosario, Universidad Autónoma de Santo Domingo (UASD), República Dominicana

José Ignacio Sánchez de Villapadierna, Instituto Cervantes - Madrid, España

Josep Seguí Dolz, Universidad Autónoma de Barcelona, España

Enric Senabre, Observatorio para la Cibersociedad, España

Guillermo Solarte Lindo, Pacifistas sin Fronteras, Colombia

Marta Torres i Vilatarsana, Observatorio para la Cibersociedad, España

Daniel Villar Onrubia, Oxford Internet Institute, Reino Unido

\title{
EDITORES ASOCIADOS
}

\author{
Lucía Patricia Carrillo Velázquez \\ Arlan Galvez Alonso \\ Ayandi Lemus Leal \\ Elena Ortiz Ballester \\ Ignacio Quintanilla \\ Beatriz Helena Rolón \\ Esteban Vázquez Cano \\ Carlos Velázquez \\ Esdras Viggiano
}




\section{Índice}

La convergencia (mediática y creativa) en la educación................................1 Juan José Sánchez Campos, Ana Almudena Jurado Torres

El modelo de administración del conocimiento MACOSC-IASC. 17 Octavio Orozco y Orozco

Aprendizaje bimodal de las competencias comunicativa y digital en contextos formales de Educación Superior: la realización procesual de Trabajos Fin de Grado en Moodle.

María Teresa Caro Valverde, María Teresa Valverde González

La brecha digital: problemas para abordar la variable socioeducativa en el estudio de la comunicación por SMS

Lucía Cantamutto

La planificación del aprendizaje digital: una propuesta innovadora a través del cine.

Carlos Monge López, Patricia Gómez Hernández

Aprendizaje ubicuo a través del teléfono móvil .85

María Pilar Quicios García, María Paz Trillo Miravalles 


\section{Table of Contents}

(Media and Creative) Convergence in the Educational Framework.....................1 Juan Jose Sanchez Campos, Ana Almudena Jurado Torres

The MACOSC-IASC Knowledge Management Model...............................17 Octavio Orozco y Orozco

Bimodal Learning of Digital and Communicative Skills within Formal Environments of Higher Education: The Procedural Elaboration of End of Degree Projects with Moodle. 43 Maria Teresa Caro Valverde, Maria Teresa Valverde Gonzalez

The Digital Gap: Problems to Address the Socio-Educational Variable in The Study of Communication via SMS.

Lucia Cantamutto

The Planning of Digital Learning: An Innovative Proposal through the

Cinema.

Carlos Monge Lopez, Patricia Gomez Hernandez

Ubiquitous Learning through the Mobile Phone. 85 Maria Pilar Quicios Garcia, Maria Paz Trillo Miravalles 


\title{
La brecha digital: problemas para abordar la variable socioeducativa en el estudio de la comunicación por SMS $^{1}$
}

\author{
Lucía Cantamutto, Universidad Nacional del Sur/ CONICET, Universidad de Deusto
}

\begin{abstract}
Resumen: El presente trabajo continúa con la discusión iniciada en investigaciones anteriores sobre los problemas inherentes a la conformación de un corpus se mensajes de texto de celular (SMS) para su estudio enmarcado en la Sociolingüística, la Sociolingüística Interaccional y la Pragmática Sociocultural. En sucesivas etapas de esta investigación, las dificultades propias de la elaboración de un corpus han sido objeto de atención: las posibilidades y fidelidad de la fuente, distinción de informantes claves, pertinencia y validez de los datos; sin embargo, para el caso particular de los SMS, se han detectado nuevas limitaciones relacionadas con el tipo de soporte donde se desarrolla esta comunicación mediada tecnológicamente. En particular, aquí nos detendremos a analizar las posibilidades del investigador a la hora de estudiar este tipo de intera cciones en relación a las características particulares de la inserción de tecnologías en los distintos estratos socioeducativos, atendiendo a las diferencias estructurales de la brecha digital en los estratos sociales.
\end{abstract}

Palabras clave: SMS, brecha digital, variable socioeducativa, sociolingüística, metodología

\begin{abstract}
This paper continues the discussion begun in previous research about the problems inherent in the creation of a corpus text messaging on mobile phones (SMS) for their study framed in Sociolinguistics, Sociolinguistics Sociocultural and Sociocultural Pragmatics. In successive stages of this research, the difficulties of the development of a corpus have been object of attention: possibilities and fidelity of source, distinction of key informants, relevance and validity of the data; however, for the particular case of SMS, we have detected new limitations related to the type of support where this technologically mediated communication develops. In particular, here we look after for analyze the possibilities of the researcher when studying this type of interactions in relation to the particular characteristics of the inclusion of technologies in the educational strata, based on the structural differences of the digital divide in the strata social.
\end{abstract}

Keywords: SMS Communication, Digital Divide, Socio-Educational Variable, Sociolinguistics, Methodology

\section{Introducción}

$\mathrm{E}$ n la segunda edición de su libro Language and the Internet, David Crystal (2006) señala que la generalidad de los sitios de internet ${ }^{2}$ nombrados en la primera publicación han desaparecido: solo cinco años separan una de otra. A la par, en su libro Everything is Miscellaneous, David Weinberger (2008: 130) augura que los sitios de internet que menciona, para marcar y compartir noticias en la web, probablemente no sobrevivan ni siquiera al tiempo que se tarda en imprimir el libro (ibid). Excepcionalmente, subraya, una comunidad de usuarios mantendrá los sitios vivos. Estos cambios y desarrollos de nuevas interfaces favorecen un desplazamiento centrífugo de las anteriores, generando, con frecuencia, una migración masiva de usuarios de una a otra. Así, el surgimiento de una nueva aplicación modifica el entorno donde se desarrollan las comunicaciones o una práctica nueva genera cambios en el esquema general de posibilidades comunicativas.

\footnotetext{
${ }^{1}$ La presente investigación se enmarca en una beca de posgrado tipo I de CONICET, en el Doctorado en Letras (Universidad Nacional del Sur) y forma parte del Proyecto de Grupo de Investigación "Estilo(s) Comunicativo(s) en la interacción para el español bonaerense: construcción de identidades, valores y creencias" (2011-2014), dirigido por la Dra. Elizabeth Rigatuso (UNS). El trabajo fue escrito durante una estancia de investigación doctoral realizada en la Universidad de Deusto, bajo la supervisión del Dr. Joseba Abaitua.

${ }^{2}$ Daniel Cassany (2012) señala que internet es algo tan cotidiano que ya no puede seguir nombrándose con mayúscula.
}

TEXTOS. Revista Internacional de Aprendizaje y Cibersociedad 
Por ejemplo, el advenimiento de las redes sociales (entre ellas, la popular Facebook) deportó a los intercambios entre amigos de los correos electrónicos y chats a su plataforma; asimismo, o la aparición de mensajería instantánea por internet en teléfonos móviles repercutió en el envío de SMS. Investigar en este campo, entonces, requiere de una importante decisión: o delimitar y recoger el corpus adecuadamente o hacer "arqueología" de fenómenos recientes.

Los diseños metodológicos para abordar las prácticas sociales y lingüísticas de las comunicaciones mediadas tecnológicamente deben atender, por tanto, a las necesidades de la investig ación y al objeto de estudio (que pueden ser complementadas con otras técnicas de recolección de corpus), ya que la mutación o desaparición es constante en estas interfaces artefactuales. Caso contrario, los resultados podrían afirman actualidades que ya son parte de prácticas del pasado ${ }^{3}$. Además, símil de tautología, no solo hace falta conocer aquello que se estudia sino los diferentes usos que se hacen sobre él: a diferencia de otros objetos de estudio, las prácticas, en y a partir de las plataformas de interacción, están pensadas para ser costumizadas según usuarios. Por tanto, si la opción no son los recorridos etnocéntricos (Fantin y Girardello 2008: 313-317), revisar cuáles son las posibilidades reales para diferentes grupos de usuarios se vuelve un imperativo (cfr. Bravo y Briz 2004). Esto se potencia aún más, desde una perspectiva de lingüística aplicada, cuando se buscan desarrollar herramientas - basadas en estas plataformas - para favorecer procesos de enseñanza en contextos reales.

Antes de adentrarnos en el tema que nos concierne, propondremos dos descargos con respecto a la problemática contemporánea en la cual inscribimos nuestra investigación. Se suele hablar de nuevas tecnologías cuando en realidad estamos mencionando un grupo heterogéneo de artefactos, dispositivos, plataformas y prácticas que ya no revisten el carácter de novedad. En tal sentido, consideramos que las aportaciones de Gobato (2013: 2014) resultan iluminadoras al respecto, llamando a este grupo “interfaces artefactuales". Para explicar esta nueva forma de denominación, Gobato (2014: 18-19) señala la necesidad de comprenderlo desde una mirada interdisciplinaria:

Las "interfaces artefactuales" se entienden acopladas problemáticamente con un conjunto de elementos, de fisonomía y estructura dispar, que pueden agruparse bajo la etiqueta de "modos de saber y comunicar". No se trata de una idea ajustada y precisa, sino más bien de una opción expresiva precaria y de fronteras porosas, utilizada a los fines de ahorrar al lector largas enumeraciones. Pueden entenderse, por un lado, como constelaciones de alternativas interaccionales pero también societales para la producción comunicativa; por otro, como constelaciones de elementos estabilizadores en los procesos de construcción de mundos sociales. Son, en suma, modalidades metodológicas de mediación, estabilización y dinamización de la experiencia.

La segunda, y consecuente, aclaración es sobre los jóvenes como grupo predilecto para estudiar los efectos de la tecnología. A esta tendencia se contrapone la visión de Morley (2012) quien duda en las investigaciones que basan su evidencia solo en el uso que hacen los jóvenes de la tecnología, porque no iluminan sobre las repercusiones en estas generaciones cuando crezcan: "los que aparentan estar cambiando los hábitos de consumo de medios están de hecho en una edad particular de sus vidas y tal vez cuando crezcan redescubran un gran placer en los viejos medios que en el presente no consideran para ellos" (ibid.).

Así, prácticas surgidas a través de y en las interfaces artefactuales están siendo abordadas en la actualidad desde diferentes disciplinas; con frecuencia, la metodología empleada es la observación participante (por ejemplo, Placencia 2010) o desde puntos ciegos, sin un trabajo específico de corpus más que lo que se releva a través del uso del propio investigador. En tal sentido, hay diversos estudios como el pionero de Christine Hine (2000), Virtual Etnography, otros más recientes como Ciberantropología. Cultura 2.0 (Vázquez Atochero, 2008) o la revisión más crítica sobre los alcances y obstáculos Digital Ethnography: an examination of the Use of New Technologies for Social Research (Murthy, 2008). Estos trabajos dan información sobre plataformas (generalmente, de

\footnotetext{
${ }^{3}$ En esta línea, podemos mencionar una larga trayectoria de trabajos sobre el chat que hacían referencia a prácticas propias del MIRC, ICQ o IRC (International Relay Chat) que fueron publicadas cuando las plataformas de interacción online habían variado notablemente: de públicas a privadas, de grupales a individuales, de redes de desconocidos a redes más íntimas y cercanas.
} 
computadoras) y su funcionamiento, así como también aspectos útiles a la hora de estudiar y comprender las diversas interfaces. Este grupo de investigaciones son exploraciones descriptivas sobre las prácticas sociales en interacción con interfaces artefactuales.

Sin embargo, uno de los aspectos más ricos para este tipo de análisis, no siempre debidamente atendido, son las entrevistas o encuestas a los propios usuarios sobre las prácticas que realizan habitualmente, sobre las expectativas y representaciones subyacentes junto a los hábitos ligados a la interacción con interfaces artefactuales (véase Murthy, 2008). Como señalamos, los estudios afines dan descripciones generalizadas desde la óptica del investigador (o con corpus recolectados solo en personas inforricas ${ }^{4}$ ), que no ofrecen información de cómo la tecnología refracta de manera disímil en la sociedad: tal el sentido de la brecha digital (Serrano Santoyo y Martínez Martínez, 2003). Precisamente, sobre esta carencia escriben Montse Vall-Lloverá, Adriana Gil-Juárez y Joel Feliú (2010).

Coincidimos con la afirmación de Murthy "Researchers should be mindful of the nuances of this divide and its social implications when representing both the remit of their work and the scope of their results" (2008: 848). Resulta evidente que las posibilidades actuales son inmensas, pero la brecha tecnológica aún persiste y, solo en la medida que consideremos las sutilezas de los fenómenos de apropiación y contacto con las nuevas tecnologías, podremos disminuir la distancia instalada en las prácticas sociales relacionadas con la tecnología.

A continuación presentaremos algunas reflexiones suscitadas a partir de nuestra experiencia de recolección del corpus, realizada durante 2011, en una ciudad de Argentina. Es probable, sin embargo, que algunas de las afirmaciones realizadas reflejen también la realidad de muchos países latinoameri$\operatorname{canos}^{5}$, donde la distribución de capitales culturales y tecnológicos es muy desigual (véase, por ejemplo sobre Brasil, Fantin y Girardello, 2008). En primer lugar, presentaremos el marco teóricometodológico al que adscribimos nuestra investigación, orientada a estudios de corte sociolinguíítico y enmarcado en la Pragmática Sociocultural. A continuación, una breve introducción a la situación actual en la escuela servirá como antecedente a las dificultades metodológicas inherentes a la recolección de un corpus de SMS en instituciones de diferente extracto socioeducativo y sociocultural. Por último, presentaremos la importancia del enfoque sociolingüístico para replantear las variables socioculturales en el estudio de la comunicación mediada digitalmente.

\section{Marco teórico-metodológico}

Esta investigación se adscribe teórica y metodológicamente a la Sociolingüística Interaccional (Gumperz, 1982 y 2001; Tannen, 1986 y 1996) e integra herramientas de Pragmática -en su vertiente Sociocultural (Bravo y Briz, 2004) y Ciberpragmática (Yus, 2010), de Análisis del Discurso (Van Dijk, 2000) y Análisis de la Conversación (Briz, 2001; Bravo y Briz, 2004; Palazzo, 2005). En estas perspectivas, el lenguaje es visto como un sistema socialmente construido y la interacción como un proceso dinámico, que los hablantes van negociando junto a la construcción de su identidad social a través de una serie de indicios lingüísticos y extralingüísticos. Estas corrientes postulan que los hablantes deducen cuáles son los conocimientos, en cada situación o evento comunicativo, que comparten y que permiten la producción y la interpretación de la interacción. En este sentido, en las perspectivas teóricas planteadas, las nociones de contexto, contexto sociocultural (Boretti, 2009), situación y competencia comunicativa (Gumperz y Hymes, 1972), competencia tecnológica (Palazzo 2011) y economía lingüística, en relación con el estilo electrónico (Vela Delfa, 2005), constituyen herramientas operativas básicas para comprender las producciones mediadas tecnológicamente.

En esta línea de análisis, en su libro sobre la metodología de investigación en sociolingüística, Moreno Fernández (1998) reitera que, para atender al uso lingüístico, es necesario estudiar la comunicación en contextos sociales y situacionales concretos; de manera tal que para verificar la incidencia de factores sociales en la variación lingüística es necesario hacer un estudio sociológico

\footnotetext{
${ }^{4}$ Corpus recolectados entre alumnos universitarios, por ejemplo, o en las redes sociales del investigador.

${ }^{5}$ Véase la plataforma Mapeo de políticas TIC en educación en Iberoamérica. Información regional sobre usos de tecnología en la educación. Disponible en http://mapeotic.org/ (fecha consulta: abril de 2014).
} 
previo en la comunidad estudiada. En general, según este autor y lo que estudios sociolingüísticos recogen, los factores sociales que más influyen en la variación lingüística son el sexo, la edad, nivel socioeducativo, nivel sociocultural, etnias. Según señala Moreno Fernández, uno de los principios inherentes a la sociolingüística es el Principio de la vinculación de los niveles sociales, es decir, el estudio de las relaciones sociales en un micro nivel deben corresponderse con el análisis de las macro estructuras sociales donde se insertan estas relaciones (ibíd., 137).

La elección de las variables que se correspondan con esto dependerá del interés particular de cada investigación. Sin embargo, Moreno Fernández presenta algunos modelos teóricos para comprender los factores influyentes que, necesariamente, variarán también de una comunidad a otra: en cada una, los indicadores de educación, ocupación, ingresos, darán diferentes estratos dependiendo de los parámetros internos de la comunidad (ibíd., 46). Entre las propuestas metodológicas para la variable clase social, Moreno Fernández recopila distintos modelos de estratificación social, entre ellos, los de Labov basados en una serie de puntos a partir de nivel de instrucción, ocupación e ingresos familiares (ibíd.). Cada investigador estratificará la sociedad en diferentes niveles según la profundidad y alcance de su estudio. Como señala Moreno Fernández, la forma de denominar esta variable altera entre clase, nivel sociocultural y nivel socioeconómico.

Por otra parte, Moreno Fernández (ibíd., 55) separa la variable nivel de instrucción, considerándola clave para la sociolingüística por cómo determina la variación lingüística. Esta variable, al igual que las demás, es compleja de delimitar y estratificar así como de equipararla entre las demás comunidades. La teoría del déficit de Bernstein, a pesar de ser muy criticada, liga el contexto cultural a la escolaridad para determinar qué tipo de código posee el hablante: restring ido o elaborado. Este punto resulta de suma importancia en nuestro análisis porque, según hemos observado en nuestra propia investigación, el nivel de instrucción no solo varía entre comunidades sino también entre escuelas. El nivel de escolarización lejos está de ser uniforme en el sistema educativo.

Este análisis, enmarcado en un estudio más amplio sobre uso y percepción de la comunicación mediada por teléfono móvil, permite relevar regularidades discursivas y fenómenos de variación pragmática y sociolingüística (Vela Delfa, 2005; Tagg, 2009), asociados a variables sociodemográficas y contextuales, en la variedad dialectal del español de Argentina, brindando una primera visión de conjunto sobre el tema. A partir de los propios avances realizados, en los cuales se focalizó en los fenómenos morfosintácticos en la comunicación por SMS, se ha verificado que los aspectos pragmáticos ofrecen particular interés en su vinculación con los rasgos propios del estilo electrónico (Vela Delfa, 2005).

En este marco, nuestro objetivo es aportar a la discusión sobre las técnicas de recolección de un corpus de mensajes de texto de teléfono móvil para su estudio sociolingüístico y, en particular, para aportar desde la lingüística aplicada al campo de las competencias comunicativas, de docentes y alumnos, en las interfaces artefactuales. En sucesivas etapas de la investigación, nos enfrentamos a decisiones metodológicas para abordar el corpus: las posibilidades y fidelidad de la fuente, distinción de informantes claves, pertinencia y validez de los datos. A partir de los avances realizados en nuestro trabajo de campo, presentamos aquí la continuación de la discusión iniciada sobre las consideraciones para establecer un corpus de SMS: por un lado, los condicionantes del soporte vinculados con características en distintos niveles de lengua (Cantamutto, 2012), en el plano de la interacción (Cantamutto, 2013b) y, por otro, la problemática de la reconstrucción de contextos y las características y operatividad variables sociolingüísticas (Cantamutto, 2013c). Asimismo, en gran parte de nuestra exploración consideramos que un aspecto importante al que atender es el dispositivo desde el que se realiza la comunicación, entendiendo la preferente vinculación entre modo de escritura (uso de abreviaciones o no, por ejemplo) y tipo de teclado (Cantamutto, 2012, 2013, 2013b y 2013c). No nos referimos con esto a "el móvil" sino a "qué móvil".

\footnotetext{
${ }^{6}$ Las diferencias entre modelos de celulares son mucho mayores que las diferencias entre ordenadores. La disposición y tamaño del teclado y de la pantalla, diccionario predictivo y modo de escritura desarrollado, facilidad para incorporar nuevas palabras, plantillas de emoticones disponibles.
} 
En particular, en la presente exposición, analizaremos las posibilidades del investigador para estudiar este tipo de interacciones, vinculado a su carácter privado e íntimo ${ }^{7}$, atendiendo a las características particulares de la inserción de tecnologías en los distintos estratos socioeducativos. De esta manera, entendemos por variable socioeducativa la complejidad propia del contexto sociocultural junto al nivel de instrucción, considerando también el nivel de instrucción de sus padres, la competencia comunicativa de los hablantes y, como novedad, el acceso real a la tecnología o competencia tecnológica. Es decir, en la situación actual de los sistemas educativos junto a las coyunturas socioeconómicas que han aumentado las diferencias sociales, la variable socioeducativa considera el entramado del universo cultural de los hablantes.

\section{Aproximaciones metodológicas al estudio de la comunicación por SMS}

A continuación, haremos una breve presentación del modo en que hemos recogido nuestro corpus, señalando las investigaciones que han servido como apoyo del proceso.

A nuestro marco teórico integramos las aportaciones sobre el análisis de la conversación tecnológica de Sanmartín Sáez (2007) y las propuestas metodológicas relativas al estudio de los SMS de Hutchby y Tanna (2008) y Tagg (2009), como principales aportes metodológicos a la investigación de los SMS. Al respecto, es necesario destacar que, además de haber un número reducido de estudios sobre la comunicación por SMS, en muchos casos, los propios investigadores señalan como "escaso" (Ling, 2005), "fortuito" (Campano Escudero 2007), sin "valor de muestra estadísticamente representativa" (Bernete, 2004) el corpus recogido para sus estudios. Otros investigadores utilizan diferentes ejercicios para relevar las características generales de este tipo de comunicación (como Pérez Rojas y Serrano Cuevas, 2006) o se detienen en cuestionarios en línea sobre actitudes, usos y preferencias (por ejemplo, Forgays, Hyman y Schreiber, 2014). En menor proporción, se advierte la existencia de un trabajo minucioso en la recolección de un número representativo de SMS, que atienda a variables de investigación, con un método sistemático de recolección, tal el caso de Hutchby y Tanna (2008), Herring y Zelenkauskaite (2009), Panckrust (2009), Tagg (2009) y Panckrust y Moïse (2012) que abordan el uso del mensaje de texto en inglés, italiano y francés.

En nuestra investigación en marcha replicamos algunos lineamientos generales propuestos por Hutchby y Tanna (2008) como técnica para la conformación de un corpus de SMS, quienes trabajaron sobre una base de 1250 SMS enviados y recibidos por dos usuarios de SMS seleccionados, transcriptos durante diferentes períodos de una semana a lo largo de seis meses del 2003. Otro aporte significativo es el de Herring y Zelenkauskaite (2009: 13), quienes recogen un corpus de 1452 SMS de la casilla de entrada (en un archivo público online) del programa de televisión italiana al que estaban dirigidos esos SMS. En el caso de Tagg (2009: 66), recogió su corpus de 10626 SMS, durante tres meses, a través de la colaboración de familiares y amigos. Por otra parte, toma de un foro público un número sustancialmente menor de SMS (441). Un procedimiento similar a este último usa Panckhurst (2009: 35) para su investigación, ya que recoge los SMS de un foro de discusión de estudiantes a distancia (que usan la modalidad del eLearning o aprendizaje en Internet).

Recientemente las posibilidades tecnológicas, sumada a la trayectoria de estudios en la materia, han dado muestras de avances dentro de la lingüística de corpus de SMS. A partir de una plataforma online www.sud4science.org, se recogieron más de 90000 SMS en la región de Languedoc-

\footnotetext{
${ }^{7}$ Dos factores más se suman a la intrincada red de decisiones que deben tomar los investigadores para estudiar la comunicación mediada digitalmente: el carácter privado y no físico de la interacción. Simula una obviedad, sin embargo, a diferencia de los estudios sobre comunicaciones orales, una de las características primarias de las interfaces artefactuales es su carácter no-físico que permite la interacción entre personas que no están coincidiendo geográficamente. En tal sentido, será necesario atender al anonimato pero también, para beneficiar la ética de la investigación, al consentimiento informado de los participantes de la comunicación. Al realizar un diseño metodológico, desestimar estos aspectos puede generar usos indebidos de información que no fue autorizada para los fines de la investigación.

${ }^{8}$ El corpus para esta investigación se conformó por teléfono, a partir de una muestra aleatoria de 2003 noruegos, quienes leyeron y deletrearon los últimos tres mensajes enviados que tenían en sus teléfonos móviles (Ling, 2005).

9 En este artículo, Campano Escudero (2007: 185) no especifica la forma de recolección de los 155 SMS "representativos".
} 
Roussillon (Panckrust y Moïse, 2012). Si bien todavía están en fase de sistematización de los datos, este colosal proyecto logró reunir, entre 2011 y 2012, su corpus gracias a colaboradores que transcribían sus SMS en la plataforma digital provista por el grupo. La mayoría de ellos completaron una encuesta sociolingüística con datos sobre edad, sexo, variedades lingüísticas (materna y bilingüismo), nivel de estudio actual, profesión. Asimismo, se recogió información sobre tipo de teléfono, paquete de mensajes contratado y representaciones sobre prácticas relacionadas al envío de SMS. Esta propuesta resulta muy interesante para el análisis de múltiples aspectos lingüísticos, tal como señalan las autoras. Sin embargo, las manifestaciones que se encuentren atenderán a una población no representativa de la sociedad. Se dejan de lado cuestiones culturales que hacen a la competencia comunicativa de los usuarios en este medio particular. Si bien este estudio podría ser replicado, con esta metodología no se atienden las variables socioeducativa ni extracto sociocultural.

Como se advierte en el anterior recorrido, algunas investigaciones recurren también a repositorios públicos de interacciones por SMS (tal el caso de Herring y Zelenkauskaite, 2009). Si bien es una forma práctica de conformar un corpus contrastivo, se está ignorando, de esta manera, la diversidad de posibilidades de conformación de díadas. En el tipo de relación que mantienen los interactuantes se sustentan también muchas de las características de la comunicación por SMS. Es posible verificar una estructura prototípica de los intercambios entre padres e hijos en el acto de habla de pedido, por ejemplo ${ }^{10}$. De manera tal que, una investigación que no atienda a las diferentes díadas, podrá sesgar aspectos de los resultados.

Nuestro diseño metodológico consiste en dos etapas, aunque la segunda no será aquí profundizada $^{11}$. La primera fase del trabajo de campo, llevada a cabo durante 2011, se realizó en dos escuelas secundarias de la ciudad de Bahía Blanca (Argentina), cuya composiciones poblacionales responden a dos estratos socioeducativos diferentes. Se trabajó con dos polos contrarios: por un lado, el grupo A fue seleccionado entre alumnos pertenecientes a la escuela que depende de la Universidad Nacional del Sur (E.M.U.N.S.) y, por otro, el grupo B, eran alumnos de la escuela de Enseñanza Media $n^{\circ} 5$, ubicada en un barrio periférico. Para acceder a la primera se requiere un examen de ingreso, mientras que en la segunda, muchas veces, se llega como parte de un sistema que excluye aún más a los excluidos.

En primera instancia, dirigimos una carta a las autoridades de ambas escuelas para que nos permitieran el ingreso a las aulas y nos contactaran con docentes del área de lengua. La recepción de la propuesta fue, desde el comienzo, contradictoria en la escuela B, donde la directora del establecimiento no demostró interés ni apoyo a la investigación. En segundo lugar se contactó a docentes interesados en participar, cediendo espacio de sus clases. Las directoras de las escuelas del grupo A indicaron cuáles eran los cursos que consideraban más apropiados para hacer el trabajo de campo, mientras que en la escuela B se trabajó con docentes conocidas.

Se realizaron entrevistas semiestructuradas para establecer la extracción socio educacional familiar y la identificación o no de los adolescentes a determinado grupo de pertenencia (bandas musicales, clubes, entre otros) o tribus urbanas. En términos generales, a todos los colaboradores se les preguntó sobre sus años de escolarización y los de sus padres, señalados en tres niveles a partir del máximo título alcanzado para poder definir el estrato socioeducativo familiar. Otros datos recabados fueron el modelo y marca de celular que utilizaban y el modo en que escribían sus SMS (letra por letra, texto predictivo ${ }^{12}$ o teclado qwerty).

\footnotetext{
${ }^{10}$ La relación entre tipos de actos de habla, relación de los interactuantes y comunicación por SMS es un aspecto que estamos trabajando en nuestra tesis doctoral.

${ }^{11}$ En la etapa actual de la investigación (2013-2015) se trabajará con una metodología de redes de amigos o familiares: se pedirá que firmen un consentimiento informado todos los participantes de las interacciones. Las redes de vínculos de amigos y familiares serán seleccionadas a partir de una distribución equilibrada de las variables de sexo, grupo etario y de nivel socio educacional. En principio, esta técnica de conformación de corpus se aplicará durante los dos años con intervalos de seis meses. La muestra definitiva se establecerá según parámetros sociolingüísticos y atenderá la región dialectal.

${ }^{12}$ La escritura predictiva es una herramienta de escritura o entrada de texto que anticipa la palabra a partir de combinar la secuencia numérica de las teclas correspondientes a dicha palabra, simplificando la escritura y disminuyendo la cantidad de teclas que se presionan (véase infra).
} 
Para la transcripción, la estrategia utilizada fue idéntica en ambas instituciones. Se solicitó a hablantes colaboradores que voluntariamente transcribieran, en papel y a partir de instrucciones precisas sobre el modo de realizarlo, los SMS enviados y recibidos en un lapso de tres días consecutivos entre sí, con especificación de las características sociolingüistas de los hablantes y de las situaciones interactivas planteadas. Las instrucciones especificaban que no debía modificarse ningún aspecto del SMS en la transcripción. Para evitar errores en la muestra se solicitó a 90 alumnos que participaran (un tercio más de la muestra recortada), permitiendo corregir el margen de error en caso de que alguien abdicara de su participación ${ }^{13}$.

Asimismo, por razones de ética, se pidió que firmaran un consentimiento informado (véase Tagg, 2009: 82), separado de las planillas de transcripción de mensajes, en el cual se aclaró a los colaboradores que podían abdicar de su participación en la investigación y negar material que consideraran demasiado privado o que pudiera afectar a terceros. De todas maneras, a fines de salvaguardar la identidad de los hablantes, se mantuvo el anonimato de la totalidad de los implicados en los intercambios así como también información de carácter privado o que pudiera perjudicar a terceros. Las planillas solo estaban identificadas con letras y números.

Dentro del conjunto de posibilidades de producción de discursos en este modo de comunicación digital hemos seleccionado los SMS intercambiados entre hablantes en el empleo cotidiano de la lengua, dejando de lado los mensajes publicitarios y aquellas producciones que son destinadas a los medios masivos de comunicación ${ }^{14}$. Sin embargo, en las planillas de transcripción se pidió a los colaboradores que transcribieran dichos mensajes que servirán para investigaciones posteriores sobre la comunicación en el ámbito institucional y, en especial, de la mercadotecnia.

Sin poder permitirnos generalizaciones sobre la situación percibida en la comunidad de estudio, podemos afirmar que existen diferencias estructurales en cuanto al uso y consumo del teléfono móvil y sus aplicaciones. Según las encuestas realizadas durante el proceso de conformación del corpus, se observó que en el grupo A casi la totalidad de los alumnos tenían teléfono móvil y habían comenzado a utilizarlo entre los 10 y 12 años. Como en Argentina recién se estaban comenzando a comercializar, solo en los dos últimos años algunos tenían Smartphone. Durante la entrevista, no se preguntó sobre el servicio tenían contratado pero, según los datos provistos por las interacciones recogidas, solo en algunos casos se señala o se excusan por la falta de crédito ${ }^{15}$.

No obstante, en el grupo B, surgieron imprevistos. Los alumnos tenían teléfonos móviles que utilizan como reproductores de música, principalmente, para tomar fotos, también, pero no para comunicarse. En las reuniones informativas, los pocos alumnos que demostraban interés en la investigación reconocían la imposibilidad de cooperar: muchos no tenían teléfono propio desde donde enviar SMS, algunos tenían un chip (que cambiaban con frecuencia) y lo colocaban en el aparato que circunstancialmente consiguieran. Hubo quienes manifestaron no tener crédito hace varios meses. De hecho, en las interacciones recogidas en este grupo, es notorio el desarrollo de conversaciones completas de personas que cargan crédito y lo usan hasta que se acaba o una frecuencia de envío y recepción de SMS distante por varios días.

\footnotetext{
${ }^{13}$ De hecho, tres de los colaboradores de 13 años informaron que no querían participar porque sus padres les negaron el permiso. En dos casos, se rompió el consentimiento informado porque los alumnos dieron sus nombres pero en un caso solamente contábamos con la clave alfanumérica, por tanto el consentimiento no fue devuelto. Por otra parte, muchos alumnos del grupo B no entregaron las planillas, lo que significó una merma significativa en la posibilidad de cruzar variables sociodemográficas.

${ }^{14}$ Este recorte se ha efectuado sobre la base de nuestros estudios previos sobre el SMS en el habla adolescente.

${ }^{15}$ Transcribimos a continuación algunos ejemplos de nuestro corpus:

Contexto: organización de encuentro entre novios. (321) AM3 a AF2 (novios): "Dale, paso cuando vos me digas. Si no me respondes antes de las 12 asumo que no tenes cred y paso 12 y 10 . Cuando llego te llamo al cel".

Contexto: pelea con el novio de la emisora. (396) AF2 a AF2 (vínculo amigas): "Anoche hable. Le dije que estaba enojada porque no me daba bola y me dijo que nada que ver y me dijo que nada que ver que no tenía crédito y nada. Sigo fastidiada pero menos porque es re lindo y me dijo cosas lindas jajaja".

Contexto: organización de actividad escolar. (898) AF1 a AF1 (vínculo amigas): "Flor me pasas lo que encontraste del laberinto? (lo de lengua) a partir de los corredores y pasillos. Si no tenes crédito llamame al fijo 4395630”.
} 


\section{Investigar en y para la escuela}

A esto se suma el contexto para el cual se están realizando las investigaciones. Los estudios cuantitativos y cualitativos sobre la distribución de los bienes tecnológicos han aportado interesantes datos sobre usos de jóvenes y docentes en diferentes países. Estas minuciosas observaciones sobre las conductas, hábitos y modos de consumo deben ser el antecedente directo a la implementación de cualquier tipo de tecnología en el aula. En tal sentido, deberían ser los mismos alumnos y docentes quienes valoren el impacto real de estas tecnologías en cada medio particular.

Lo paradójico es que, a pesar de que las interfaces artefactuales son consideradas como herramientas útiles para democratizar la educación y reducir las diferencias sociales (Sunkel, 2006), sucede que los bretes suelen acrecentarse: la brecha tecnológica no se define solo por la adquisición de determinado dispositivo (Fantin y Girardello, 2008: 323; Massana Molera, 2011). Por el contrario, y a pesar de que los gobiernos apuestan a disminuir este resquicio con programas que provean a los alumnos igualdad de oportunidad al acceso a la tecnología, las diferencias en infraestructura, usos, consumos, hábitos siguen marcando pautas de exclusión social. La bibliografía sobre este tema señala, consecuentemente, la necesidad de políticas públicas que provean, como sucedió en algunos países, acceso a internet de banda ancha de bajo costo o gratuito, para el desarrollo de competencias en todos los usuarios.

La escuela se debate entre reproducir los modelos sociales obsoletos o reflejar los cambios sociales del presente. Ante esta premisa, la incorporación de diversas tecnologías no deja de replantear el mismo problema: ¿subsidiarias o revolucionarias? ¿Significativas o no? (Vacca, 2011). Muchas discusiones que se dieron en torno al programa Conectar Igualdad ${ }^{16}$, que se desarrolla en Argentina desde el año 2010, tenían su origen en diferentes núcleos temáticos: posibilidades reales de disminución de la brecha digital, competencias y habilidades de docentes y de alumnos, contenidos a impartir a través y a partir de las netbooks. Los periódicos, probablemente, no reflejaron el ambiente inicial que al interior de las instituciones educativas se vivió. Sin embargo, muchas son las visiones optimistas sobre la netbook y su mejora en el ánimo de los destinatarios directos: los alumnos, mientras que hay quienes señalan el bajo impacto que tuvo, en gran parte, debido al "analfabetismo digital" (Sigal, 2011).

En tanto, las mejoras tecnológicas - tales como desarrollo de dispositivos, mejora de software-, no son las que favorecen la inclusión, sino que es necesaria la distribución social y geográfica de sus capacidades. Recientes investigaciones auguran que la disponibilidad de internet en los teléfonos móviles reducirá la brecha digital en Latinoamérica (véase Jordán, Galperín y Peres, 2010). Sin embargo, el potencial de esta afirmación ignora, por ejemplo, que la conectividad de los teléfonos móviles descansa en el alcance de las antenas y éstas lejos están de ser omnipresentes. Estas antenas suelen ser rechazadas por la comunidad donde se instalan pero reclamadas por los marginados geográficamente de sus conos de conectividad. Por tanto, la presencia de un dispositivo de comunicación no es condición suficiente para garantizar la conectividad, menos aún la comunicación. Nuevamente: una división centro-periferia que se traduce, en otras palabras, en infopobres o inforri$\cos$ (en relación al acceso que tengan a la información en los nuevos medios, es decir, a partir de su accesibilidad a las nuevas tecnologías) o, aún más, en residentes o usuarios de las nuevas tecnologías (Quevedo, 2012). Marginados, socialmente, tecnológicamente ${ }^{17}$.

De esta manera, atendiendo a que el objeto a largo plazo de nuestra investigación sobre comunicación por SMS es poder desarrollar herramientas para la enseñanza de la lengua, la opción más honesta es atender al mayor universo existente de actores sociales en las instituciones educativas.

Cuando observamos estadísticas sobre el uso de dispositivos móviles, notaremos con frecuencia esta contradicción que acompaña los supuestos sobre la omnipotencia del móvil: a pesar de verificarse una penetración mayor al 100\% en más de cien países (ITU Stat Shot, 2012), esta cifra esconde la

\footnotetext{
16 http://www.conectarigualdad.gob.ar/seccion/sobre-programa-6 (fecha de consulta: febrero de 2014).

${ }^{17}$ Un artículo reciente señala la aparición de una nueva clase social marginada: aquellos que no dejan Big Data y que son excluidos de esta revolución: como señala su autora, es necesario considerar las huellas de aquellos que las hacen más débiles (Lieber, 2013).
} 
distribución real de esas líneas. En el mismo informe, señalan que en los países en crecimiento el $87 \%$ de las suscripciones son por pre-pago y que el $75 \%$ de su población no está usando internet. Si estos datos los confrontamos, por ejemplo, con las estadísticas oficiales de Argentina, corroboraremos que hay una penetración mayor al $100 \%$ que surge de dividir el número de tarjetas SIM en el mercado por la cantidad de habitantes del país. Sin embargo, las llamadas mensuales realizadas significan un 6\% de las líneas en uso, realizando una llamada cada teléfono móvil. Al respecto, coincide el informe de Naciones Unidas sobre las tecnologías móviles (2012): "suscriptions do not reflect actual owership (...) Mobile penetration is based on SIM cards sold".

Por otra parte, sumado a la división digital entre ambos grupos escolares, se encuentra la disímil predisposición para participar en la investigación. El primer obstáculo con el que nos enfrentamos con el grupo B fue la aceptación de la actividad. No valió, siquiera, darles tiempo durante la clase para que lo realizaran. La cultura escolar de este grupo está ligada a prácticas de confrontación constante con el docente, falta de voluntad para participar en actividades escolares y desafío permanente a la institución. Así, los resultados no fueron los previstos: la recolección del corpus no atendió en su totalidad a los estratos socioeducativos. A diferencia del grupo A, que realizó con presteza la transcripción (sin omitir información y haciendo aclaraciones pertinentes) y devolución de las planillas, en el grupo B se recolectaron cerca del 10\% de las planillas entregadas. Estos jóvenes que asisten desmotivados a la escuela requieren un abordaje diferenciado. La muestra definitiva, por tanto, no atendió a todos los parámetros previstos en la investigación. De esta manera, el supuesto subyacente sobre el uso, percepción y representación de los dispositivos móviles no podía aplicarse a ambos grupos de manera homogénea.

Aún más, hoy en día, se suma otra dificultad: en entrevistas auxiliares recientes, los docentes observan que los alumnos de escuelas periféricas no suelen tener teléfonos $\mathrm{y}$, menos aún, tener teléfonos con internet. Al contrario, en las escuelas de mayor nivel socioeducativo, el SMS está siendo desplazado por las plataformas de interacción tipo Whatsapp, mensajes de Facebook, Skype para el teléfono y otro repertorio de servicios de mensajería que usan internet. De esta manera, se replica aquello que se observa a nivel macro: los países centrales van adecuándose paulatinamente a las tecnologías mientras que los países periféricos lo hacen en saltos; así, en los diferentes grupos sociales, ocurre lo mismo. Difícilmente, la alfabetización digital y la literacidad digital de quienes llegan "por saltos" a los cambios en las interfaces sea igual que entre quienes van experimentando con la tecnología y apropiándose de ella de manera gradual. Nuevamente, además, aquellos que son la clase invisible en la Big Data, no arrojarán información sobre lo que desean, usan y rechazan.

\section{A modo de cierre}

Digital inclusion must include social, cultural, technological, and intellectual dimensions, in order to favor forms of belonging and assure the effective participation of people in the culture. (Fantin y Giardello, 2008: 323)

A lo largo del trabajo hemos expuesto algunas de las dificultades de orden metodológico que están en estrecha relación con la variable socioeducativa y sociocultural. Nuestro objetivo inicial era alertar no solo sobre estas sutilezas del objeto de estudio en particular sino también de la necesidad de responder a estas diferencias a la hora de elaborar herramientas didácticas o dispositivos pedagógicos para implementarse en el aula. En tal sentido, las investigaciones deben buscar la manera de reflejar las condiciones, los usos y las percepciones de los diferentes actores sociales a los que los avances tecnológicos tocan de manera desigual. Consideramos que el enfoque sociolingüístico junto a la Pragmática Sociocultural, ofrecen herramientas válidas para realizar investigaciones que puedan mejorar los resultados no solo de propuestas del campo de la lingüística aplicada sino también en las políticas públicas sobre la inclusión digital.

Por tanto, si el objetivo último es disminuir la brecha digital o tecnológica y, al mismo tiempo, favorecer los procesos de democratización de la educación y aumentar el impacto positivo de los programas de inclusión digital, las investigaciones que sustenten todo este entramado necesariamente 
deberán comenzar a reparar en los pequeños resquicios donde las diferencias se ocultan. En particular, aquellas investigaciones que deseen dar cuenta de los procesos de apropiación de los usuarios/hablantes, necesariamente, deberán ahondar en qué recorrido hicieron los diferentes grupos sociales en relación a la tecnología. Aún más: en esta distinción cabe desgranar este sustantivo: el acceso a la tecnología es el acceso a dispositivos y aparatos, disposición de infraestructura para la conectividad, educación en literacidad digital, competencias tecnológicas y comunicativas, entre otras. Cada uno de estos factores repercute, de diferente manera, en el uso particular de los usuarios de los diferentes estratos socioeducativos y en su correlato con la brecha digital. 


\section{REFERENCIAS}

Bernete, F. (2012). "Las preocupaciones y averiguaciones que se manifiestan en torno al lenguaje juvenil usado en los móviles e Internet". Revista de Estudios para el Desarrollo Social de la Comunicación 1(6): 197-213.

Boretti, S. (2003). "Tests de hábitos sociales y la investigación de la cortesía". Actas del Primer coloquio del programa EDICE "La perspectiva no etnocentrista de la cortesía: identidad sociocultural de las comunidades hispanohablantes”, Bravo, Diana (ed.), pp. 198-202, Stockholm, Stockholm University.

Bravo, D. (2005). Estudios sobre la (des)cortesía en español. Estocolmo/Buenos Aires: Dunken.

Bravo y Briz, Antonio (eds.) (2004). Pragmática sociocultural: Estudios sobre el discurso de cortesía en español. Barcelona: Ariel.

Briz, A. (2001). El español coloquial en la conversación. Barcelona: Ariel.

Campano Escudero, B. (2007). "Análisis lingüístico-pragmático de un corpus de mensajes SMS". Ferrán 8: 185-210. Disponible en: www.educa.madrid.org/web/ies.jaimeferran. colladovillalba/revista2 (fecha consulta: marzo 2011).

Cantamutto, L. (2012). "El uso de los mensajes de texto en el habla adolescente del español bonaerense". Tesis de Grado. Bahía Blanca: UNS.

- $\left(2013^{\mathrm{a}}\right)$. "Ok, cortado de mierda:\$". Las fórmulas de tratamiento como recurso expresivo en la interacción por SMS". IV Coloquio de Investigadores en Estudios del Discurso, Buenos Aires: ALED (trabajo en evaluación).

- (2013b). "La recursividad de las interacciones contemporáneas. Límites teórico-metodológicos del estudio de los SMS como conversación”. Revista de Ciencias Sociales de la UNQ, $2^{\circ}$ época, 23: 83-103.

- (2013c). "La conformación de un corpus de mensajería de texto: la interacción mediada digitalmente". Actas de las IV Jornadas de Investigación de Humanidades. Homenaje a Laura Laiseca, Gambón, Lidía (2013), Bahía Blanca, Universidad Nacional del Sur.

Crystal, D. (2006). Language and the Internet. Cambridge: Cambridge University Press.

Fantin, M. y Girardello, G. (2008). "Digital Literacy and Cultural Mediations to the Digital Divide." En Digital Literacy: tools and methodologies for Information Society, Rivoltella, P. C., 5174. EEUU/England: IGI Publishing.

Forgays, D. K., Hyman, I. y Schreiber, J. (2014). "Texting everywhere for everything: Gender and age differences in cell phone etiquette and use." Computers in Human Behavior 31:314-321.

Gobato, F. (2013). "La interacción social en la comunicación contemporánea". Revista de Ciencias Sociales de la $U N Q, 2^{\circ}$ época, 2: 49-70.

- (2014). La comunicación de la comunicación. Oralidad, escritura y digitalización en la interacción contemporánea. Bernal: Universidad Nacional de Quilmes.

Gumperz, J. (ed.). (1982). Language and Social Identity. Cambridge: University Press.

Gumperz, J. y Hymes, D. (eds.). (1972). Directions in sociolinguistics: The ethnography of communication. New York: Holt, Rinehart and Winston.

Hard af Segerstad, Y. (2002). "Use and Adaptation of the Written Language to the Conditions of Computer-Mediated Communication.” Tesis de doctorado, University of Goteborg. Institutionen för lingvistik University of Gothenburg Faculty of Arts. Department of Linguistics.

Herring, S. y A. Zlenkauskaite (2009). "Symbolic Capital in Virtual Heterosexual market.” Written Communication 26.1: 5-31. Disponible en: www.sagepublications.com (fecha consulta: marzo 2010).

Hine, C. (2000). Virtual ethnography. Sage Publications.

Hutchby, I. y V. Tanna. (2008). “Aspects of sequential organization in text message Exchange.” Discourse \& Communication 2(2): 143-164. Disponible en: www.sagepublications.com (fecha consulta: marzo 2010). 
Lieber, J. (2013). “A new underclass: the people who Big Data leaves behind.” Disponible en: http://www.fastcoexist.com/3017102/a-new-underclass-the-people-who-big-data-leavesbehind?goback=.gde_91073_member_274253965\#\%21 (fecha consulta: noviembre de 2013).

Ling, R. (2002). "Chicas adolescentes y jóvenes adultos varones: dos subculturas del teléfono móvil”. Revista de estudios de juventud 57: 33-46.

Massana Molera, E. (2011). "La brecha digital generacional en el uso de móviles en Cataluña”. En m-Todos, tendencias y oportunidades de la movilidad digital, García Medina, I. y Contreras-Espinosa, R. S. España: Universidad de Vic.

Moreno Fernández, F. (1998). Principios de sociolingüística y sociología del lenguaje. Barcelona: Ariel.

Morley, D. (2012). Entrevista realizada por Igarzábal, B. (2012), Clase ${ }^{\circ} 7$ del Módulo I, del Curso de Posgrado Gestión Cultural y Nuevas Tecnologías. Argentina: FLACSO.

Murthy, D. (2008). "Digital Ethnography an Examination of the Use of New Technologies for Social Research." Sociology 42(5): 837-855.

Palazzo, M. G. (2005). “¿Son corteses los jóvenes en el chat? Estudio de estrategias de interacción en la conversación virtual". Revista Textos de la Cibersociedad 5. Disponible en: http://www.cibersociedad.net (fecha consulta: junio 2011).

- (2011). "La expresión de la subjetividad en el ciberespacio: prácticas discursivas en blogs de jóvenes". Actas del V Coloquio de Investigadores en Estudios del Discurso. Disponible en: www.unvm.edu.ar/archivos/jornada_discurso/PALAZZO.pdf (fecha consulta: marzo 2012).

Panckhurst, R. (2009). "Short Message Service (SMS): typologie et problemàtiques futures". En Pholyphonies, pour Michelle Lanvin, Arnaviellie, T. (coord), 33-52. Université PaulValery Montpellier.

Panckhurst, R. y Moïste, C. (2012). "French text messages From SMS data collection to preliminary analysis". Lingvisticae Investigactiones 35(2): 289-317.

Pérez Rojas, E. y Serrano Cuevas, Y. (2006). Impacto de los mensajes de texto en el uso del lenguaje escrito: en los alumnos de 8vo y 9no grado de la U. E. Colegio de Nuestra Señora de Fátima, Mérida. Disponible en: http://tesis.ula.ve/pregrado/tde_busca/arquivo.php?cod Arquivo=1033 (fecha consulta: mayo 2011).

Placencia, M. (2010). "Yahoo! Respuestas como columna de consejos: algunos rasgos de un género híbrido". Tonos Digital 20. Disponible en: http://www.tonosdigital.es/ojs/index.php/tonos/ article/view/601/459 (fecha consulta: octubre 2013).

Quevedo, L. A. (2012). "Los medios de comunicación en la era de las TIC". Clase 1, Maestría en Gestión Cultural y Comunicación. Buenos Aires: FLACSO.

Sanmartín Sáez, J. (2007). El chat: la conversación tecnológica. Madrid: Arco/libros.

Santoyo, A. S. y Martínez, E. M. (2003). La brecha digital: mitos y realidades. México: UABC.

Sigal, P. (2011). "Generación 2.0: nacieron y crecieron con la PC, pero no llegan a aprovecharla", Diario Clarín, 20 de julio de 2011. http://www.clarin.com/educacion/Nacieron-crecieronPC-1legan-aprovecharla_0_523147797.html (fecha consulta: octubre 2013).

Sunkel, G., (2006). Las tecnologías de la información y la comunicación (TIC) en la educación en América Latina. Una exploración de indicadores. Santiago de Chile: CEPAL.

Tagg, C. (2009). A corpus linguistics study of SMS text messaging. United Kingdom: University of Birmingham. Disponible en: http://etheses.bham.ac.uk/253/1/Tagg09PhD.pdf (fecha consulta: junio 2012).

Tannen D. (1996). Género y discurso. Barcelona: Paidós.

- (1986). Coherence in spoken and written discourse. Vol. XII. New Jersey: ABLEX.

Terceiro, J. B. y Matías, G. (2001). Digitalismo. El nuevo horizonte cultural. Madrid: Taurus digital.

Vacca, A. M. (2011). "Criterios para evaluar proyectos educativos de aula que incluyen al computador". Revista Iberoamericana de Evaluación Educativa 4(2).

Vall-Llovera, M., A. Gil-Juárez y J. Feliú. (2010). "La interpretación de los jóvenes ante sus prácticas tecnológicas". Revista Argentina de Estudios de Juventud 3. Disponible en: http://www.perio.unlp.edu.ar/revistadejuventud/?q=node/55 (fecha consulta: junio 2011).

Vázquez Atochero, A. (2011). Ciberantropología. Cultura 2.0. España: Editorial UOC. 
Vela Delfa, C. (2005). "El correo electrónico: un nuevo género en nacimiento". Tesis Doctoral, Universidad Complutense de Madrid. Disponible en: http://www.galanet.be/publication /fichiers/tesis_cristina_vela_delfa.pdf (fecha consulta: marzo 2012).

Weinberger, D. (2008). Everything is Miscellaneous, The Power of the New Digital Disorder. New York: Henry Holt and Co.Inc.

Yus, F. (2010). Ciberpragmática 2.0. Nuevos usos del lenguaje en internet. Barcelona: Ariel.

\section{Informes}

Informe Naciones Unidas (2012). Mobile techonology and empowering. Disponible en: http://www. undpegov.org/sites/undpegov.org/files/undp_mobile_technology_primer.pdf (fecha consulta: octubre 2013).

ITU STAT SHOT (2012). El mundo de las tecnologías. Disponible en: http://www.youtube.com/ watch?v=HZcHwQ6tL2k (fecha consulta: octubre 2013).

Mapeo de políticas TIC en educación en Iberoamérica. Información regional sobre usos de tecnología en la educación. Disponible en: http://mapeotic.org/ (fecha consulta: abril de 2014).

\section{SOBRE LA AUTORA}

Lucía Cantamutto: Licenciada y Profesora en Letras por la Universidad Nacional del Sur (Argentina) con estudios sobre Comunicación y Sociología en la Universidad Autónoma de Aguascalientes (México). La tesis de licenciatura fue sobre "El uso de los mensajes de texto en el habla adolescente del español bonaerense". Actualmente, es becaria doctoral del Consejo Nacional de Investigaciones Científicas y Técnicas (Argentina) para realizar su tesis en el Doctorado en Letras: "Economía y expresividad lingüísticas: aspectos pragmáticos de la comunicación por SMS en español bonaerense" y realizó una estancia de investigación en la Universidad de Deusto (2013-2014), en el marco del programa Erasmus Mundus Action 2, Europlata. 